\title{
Engaging patients and families to create a feasible clinical trial integrating palliative and heart failure care: results of the ENABLE CHF-PC pilot clinical trial
}

Marie Bakitas ${ }^{1,2^{*}}$ (D) J. Nicholas Dionne-Odom², Salpy V. Pamboukian ${ }^{3}$, Jose Tallaj ${ }^{3}$, Elizabeth Kvale ${ }^{4}$, Keith M. Swetz ${ }^{4}$, Jennifer Frost ${ }^{5}$, Rachel Wells ${ }^{6}$, Andres Azuero ${ }^{2}$, Konda Keebler ${ }^{2}$, Imatullah Akyar ${ }^{2,7}$, Deborah Ejem²,

Karen Steinhauser ${ }^{8}$, Tasha Smith ${ }^{2}$, Raegan Durant ${ }^{9}$ and Alan T. Kono ${ }^{5}$

\begin{abstract}
Background: Early palliative care (EPC) is recommended but rarely integrated with advanced heart failure (HF) care. We engaged patients and family caregivers to study the feasibility and site differences in a two-site EPC trial, ENABLE CHF-PC (Educate, Nurture, Advise, Before Life Ends Comprehensive Heartcare for Patients and Caregivers).

Methods: We conducted an EPC feasibility study (4/1/14-8/31/15) for patients with NYHA Class III/IV HF and their caregivers in academic medical centers in the northeast and southeast U.S. The EPC intervention comprised: 1) an in-person outpatient palliative care consultation; and 2) telephonic nurse coach sessions and monthly calls. We collected patient- and caregiver-reported outcomes of quality of life (QOL), symptom, health, anxiety, and depression at baseline, 12- and 24-weeks. We used linear mixed-models to assess baseline to week 24 longitudinal changes.
\end{abstract}

Results: We enrolled 61 patients and 48 caregivers; between-site demographic differences included age, race, religion, marital, and work status. Most patients (69\%) and caregivers (79\%) completed all intervention sessions; however, we noted large between-site differences in measurement completion (38\% southeast vs. 72\% northeast). Patients experienced moderate effect size improvements in QOL, symptoms, physical, and mental health; caregivers experienced moderate effect size improvements in QOL, depression, mental health, and burden. Small-to-moderate effect size improvements were noted in patients' hospital and ICU days and emergency visits.

Conclusions: Between-site demographic, attrition, and participant-reported outcomes highlight the importance of intervention pilot-testing in culturally diverse populations. Observations from this pilot feasibility trial allowed us to refine the methodology of an in-progress, full-scale randomized clinical efficacy trial.

Trial registration: Clinicaltrials.gov NCT03177447 (retrospectively registered, June 2017).

Keywords: Heart failure, Palliative care, Caregiver, Telehealth, Rural, Intervention development

\footnotetext{
* Correspondence: mbakitas@uab.edu

${ }^{1}$ School of Nursing and Department of Medicine, Division of Gerontology,

Geriatrics, and Palliative Care, University of Alabama at Birmingham,

Birmingham, AL, USA

${ }^{2}$ School of Nursing, University of Alabama at Birmingham, 1720 2nd Ave

South, MT 412C, Birmingham, AL 35294, USA

Full list of author information is available at the end of the article
} 


\section{Background}

Of the 6 million U.S. individuals with heart failure (HF), approximately 300,000 will die each year. By 2030, HF prevalence is expected to swell by $46 \%$ to over 8 million [1]. Evidence-based advances in medication management (e.g. ACEIs, beta-blockers), coronary revascularization, mechanical circulatory support devices, and cardiac resynchronization have lengthened $\mathrm{HF}$ survival [2, 3]. However, these added years of life are often accompanied by significant morbidity and prognostic uncertainty requiring difficult discussions and decisions about treatments and quality of life (QOL) $[2,4,5]$. These burdens often extend beyond the individual with $\mathrm{HF}$ to family members who must take on new roles to assist patients with disease management [6]. These caregivers can develop needs [7] that, when unmet, lead to psychological stress and poorer health $[8,9]$.

Recent professional guidelines recommend involvement of palliative care for New York Heart Association (NYHA) Class III/IV and American Heart Association (AHA) Stage C/D HF patients undergoing advanced therapies, facing difficult medical decisions, having complex or refractory symptoms, and having overstrained caregivers [10-15]. Yet only 1-out-of-3 HF patients receive palliative care and usually not until the final weeks to days before death [16]. Therefore, integrating palliative care earlier in the HF trajectory, when patients are relatively healthy and functional, may help patients and their families cope and live better with advanced disease [17-20].

High quality trials in cancer have demonstrated positive patient and family caregiver outcomes from early palliative care (EPC) [21, 22]. However, given the difficulties in prognostication, the prevalence of sudden cardiac death, [23] and an erratic illness trajectory, [24] it is not clear when or how to integrate palliative care in HF [25]. Furthermore, trials of EPC have rarely included persons with low income and education, of a minority race, and who reside in rural, medically-underserved areas [25-29]. Thus, it is imperative to develop models of EPC that are responsive to the HF trajectory, and also are tailored to be culturally appropriate for minority and underserved populations for whom HF can have particularly pernicious effects.

To address these challenges, we actively engaged patients and family members with diverse socioeconomic and racial backgrounds to aid in further refining and culturally-tailoring ENABLE CHF-PC (Educate, Nurture, Advise, Before Life Ends Comprehensive Heartcare for Patients and Caregivers), a telephonic EPC intervention for rural-dwelling, underserved HF patients and their family caregivers. In a proof-of-concept, formative evaluation study [30], we translated materials and protocols from our successful EPC ENABLE oncology model [31-33] to a HF population. This study demonstrated acceptability, feasibility, and a signal of potential efficacy in an educationally, socioeconomically, and racially homogeneous sample of 11 patient-caregiver dyads [30] Thus, the current ENABLE CHF-PC feasibility trial was expanded to include an additional site in the southeastern U.S. that had greater racial and cultural diversity in order to identify intervention acceptability and feasibility and thus greater generalizability to a broader U.S. population. The purpose of this study was to: 1) determine the feasibility of recruiting and retaining a rural, racially-diverse sample of patient-caregiver dyads for 24 weeks and 2) explore longitudinal patient and caregiver outcomes including QOL, global health, anxiety, and depression to inform intervention measures and the need for additional protocol modifications for a larger clinical efficacy trial.

\section{Methods}

\section{Study design}

In this feasibility study, conducted April 1, 2014 to December 31, 2015 individuals with AHA Stage C/D and/or NYHA Class III/IV HF and their family caregivers received the ENABLE CHF-PC intervention and were followed for 24 weeks. The study protocol was approved by the institutional review boards of Dartmouth College (Lebanon, New Hampshire) and the University of Alabama at Birmingham (Birmingham, Alabama) and all participants provided written informed consent.

\section{Setting and sample}

Study participants were recruited from cardiology clinics at 1) Dartmouth-Hitchcock Medical Center (DHMC), Lebanon, $\mathrm{NH}$, which serves a largely rural, white population in a state ranked lowest in religiosity, and 2) the University of Alabama at Birmingham (UAB), Birmingham, $\mathrm{AL}$, which serves a diverse rural-urban population that includes a large proportion of Blacks/African-Americans in a state ranked highest in religiosity [34]. Study coordinators at both sites reviewed outpatient cardiology clinic schedules to identify eligible patients. Following physician approval, a study coordinator approached patients and their caregivers during a clinic appointment to explain the study and obtain consent.

Patient inclusion criteria were: 1) diagnosis of NYHA Class III/IV and/or AHA Stage C/D HF; 2) English speaking; 3) $\geq 50$ years of age; and 4) completion of baseline questionnaires. Exclusion criteria were: 1) dementia or impaired cognition (Callahan score $\leq 4$ ) [35], 2) active Axis I psychiatric or substance use disorder; and 3) noncorrectable hearing impairment. Patients were asked to nominate a caregiver for participation, defined as "someone who knows you well and is involved in and has knowledge of your medical care." Caregivers were only excluded for non-correctable hearing loss. 


\section{The ENABLE CHF-PC intervention}

A comprehensive description of the evolution and development of ENABLE CHF-PC has been described in detail elsewhere [30]. Briefly, the ENABLE CHF-PC intervention (Fig. 1) tested in this study included: 1) an in-person outpatient palliative care consultation (caregiver invited to attend) following National Consensus Guidelines [36], 2) weekly, semi-structured palliative care nurse coach (patients: 6 sessions; caregivers: 4 sessions) telephone and monthly follow-up sessions using Charting Your Course, an educational guidebook. Sessions, conducted weekly, covered the following topics problem solving, self-care, symptom management, decision-making and advance care planning, and life review (patients only) that were tailored to individual participant needs. The life review sessions were based on Steinhauser and colleagues' Outlook intervention [37]. The goal of the sessions was to encourage participants to feel empowered and to develop skills that would assist them to make value-driven decisions about their medical and life-sustaining treatment choices as their disease worsened: Patients and caregivers were assigned separate nurse coaches to increase their sense of confidentiality.

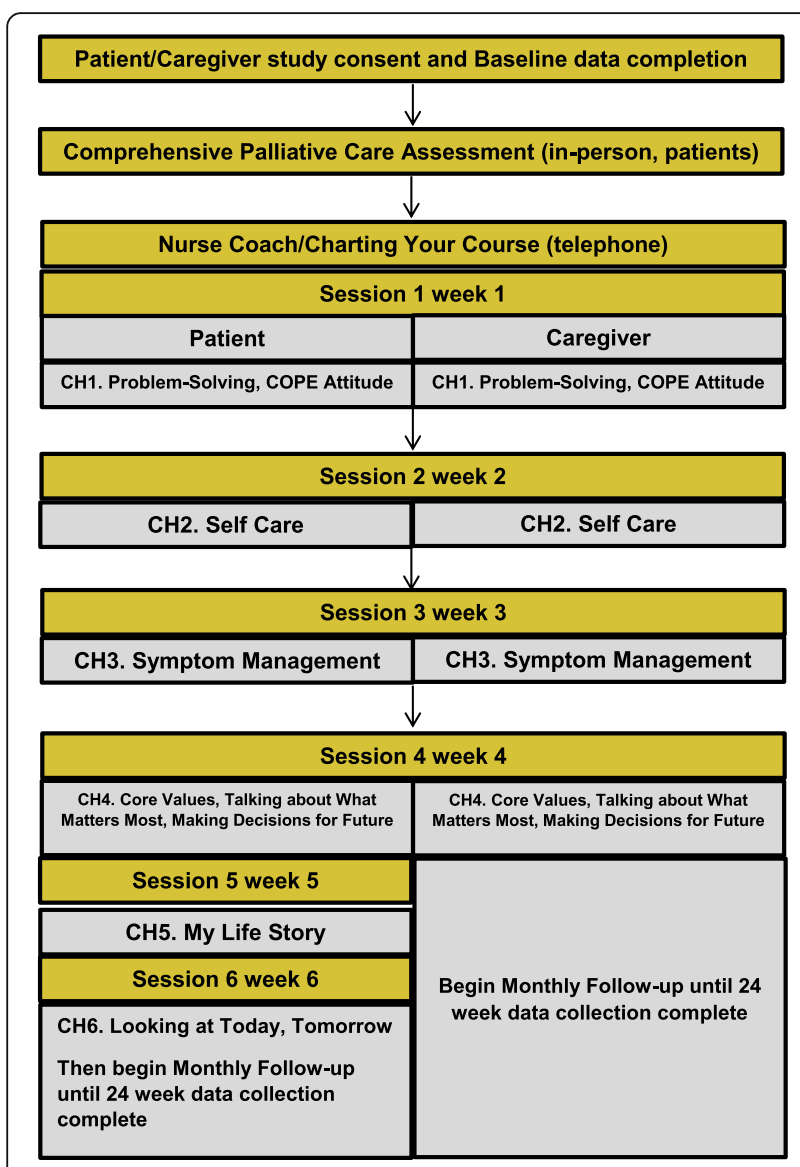

Fig. 1 Study Schema
Five nurse coaches each received $20 \mathrm{~h}$ of training including self-study of intervention protocols and scripts and interactive role-play of 10 digitally-recorded practice sessions. The nurse coaches were debriefed on their training sessions by the principal investigator (PI) (MB) and co-investigator (co-I) (JND-O) and were provided with constructive feedback. Intervention fidelity was maintained through standardized training, the use of structured interventionist scripts, use of standardized session documentation templates, and weekly PI and co-I supervisory team meetings [38].

\section{Data collection and measures}

Study coordinators completed measures with patients and caregivers by phone at baseline, 12- and 24-weeks. Participants received a $\$ 10$ check for each completed measurement occasion. Baseline demographics included age, gender, race/ethnicity, religion, marital and work status, educational level, and medical insurance. Clinical characteristics abstracted from electronic health records included NYHA class, ejection fraction, presence of an implanted heart device, medications, and laboratory data. These data were entered into the Seattle Heart Failure Model (SHFM) web-based calculator to compute 1, 2 and 5-year survival estimates (https://depts.washington.edu/shfm/). Nurse coaches also informed patients and caregivers that the purpose of this pilot trial was to determine intervention and study procedure acceptability in a new patient population (those with heart failure from diverse socioeconomic cultures). Hence the nurse coach would be actively seeking their critique and feedback throughout the intervention in order to make improvements for future patients. Nurses recorded sessions, and actively tracked patient and caregiver feedback on intervention components that were found to be helpful or in need of improvement in a Research Electronic Data Capture (REDCap) database [39]. Additional file 1: Table S1 shows patient- and caregiverreported outcome measures.

\section{Statistical analysis}

The feasibility primary aim was determined by monitoring participants' study status (enrolled, deceased, lost to follow-up) and calculating intervention and measurement completion rates (e.g. actual \# completed/possible \# per protocol). Patient and caregiver demographic characteristics were tabulated and compared between sites with bivariate tests of association and effect sizes (Cohen's $d$ [40] or $d$-equivalent [41] or nominal variables). We assessed associations between baseline characteristics and participant attrition using simple logistic regressions. We used estimated odds ratios to determine associations between patient characteristics and attrition. 
We used longitudinal, fitted, linear mixed methods, adjusted for covariates associated with attrition, to estimate participant-reported outcomes' changes from baseline to follow-up (12 and 24 week means combined) [42]. Change estimates were transformed to effect sizes (Cohen's $d$ ) using baseline estimates of pooled standard deviations. Change was estimated overall and by site. All analyses were conducted using SAS v9.4.

Due to the exploratory nature of the study, we relied on effect size estimation using Cohen's guidelines for magnitude of effect size $d$ (i.e. small: 0.2, moderate: 0.5, and large: 0.8 ) rather than hypothesis testing to interpret results; however we also report $p$-values for completeness.

\section{Results}

\section{Sample characteristics}

We assessed 431 patients for eligibility (Fig. 2); approached 120 eligible patients for participation; and enrolled 61 patients (50\% response rate) and 48 family caregivers. Eligible patients declined participation due to "not interested" $(n=22)$ or "not needed" $(n=8)$.

Overall, patients $(n=61)$ were a mean age of 71 years, male $(51 \% ; n=31)$, white $(80 \%, n=49)$, Protestant $(65.6 \%, n=40)$, married or living with a partner $(62 \%, n$ $=38)$, retired $(56 \%, n=34)$, were high school or General Education Diploma (GED) graduates $(43 \%, n=26)$ had Medicare/private insurance $(64 \%, n=39)$ and were rural $(72.1 \%, n=44)$ (Table 1). Patients and caregivers lived a median of 46 miles (range 1-177 miles) from UAB and 54 miles (range 6-128 miles) from DHMC. Compared to DHMC, UAB patients had higher proportions of black, Protestant, never married, and patients on disability. Clinically, most patients were NYHA Class IIIa/b or IV, with a mean ejection fraction of $38 ; 46 \%$ had no implanted cardiac device; $80 \%$ were on beta-blockers; and $59 \%$ were on statins. SHFM survival probabilities

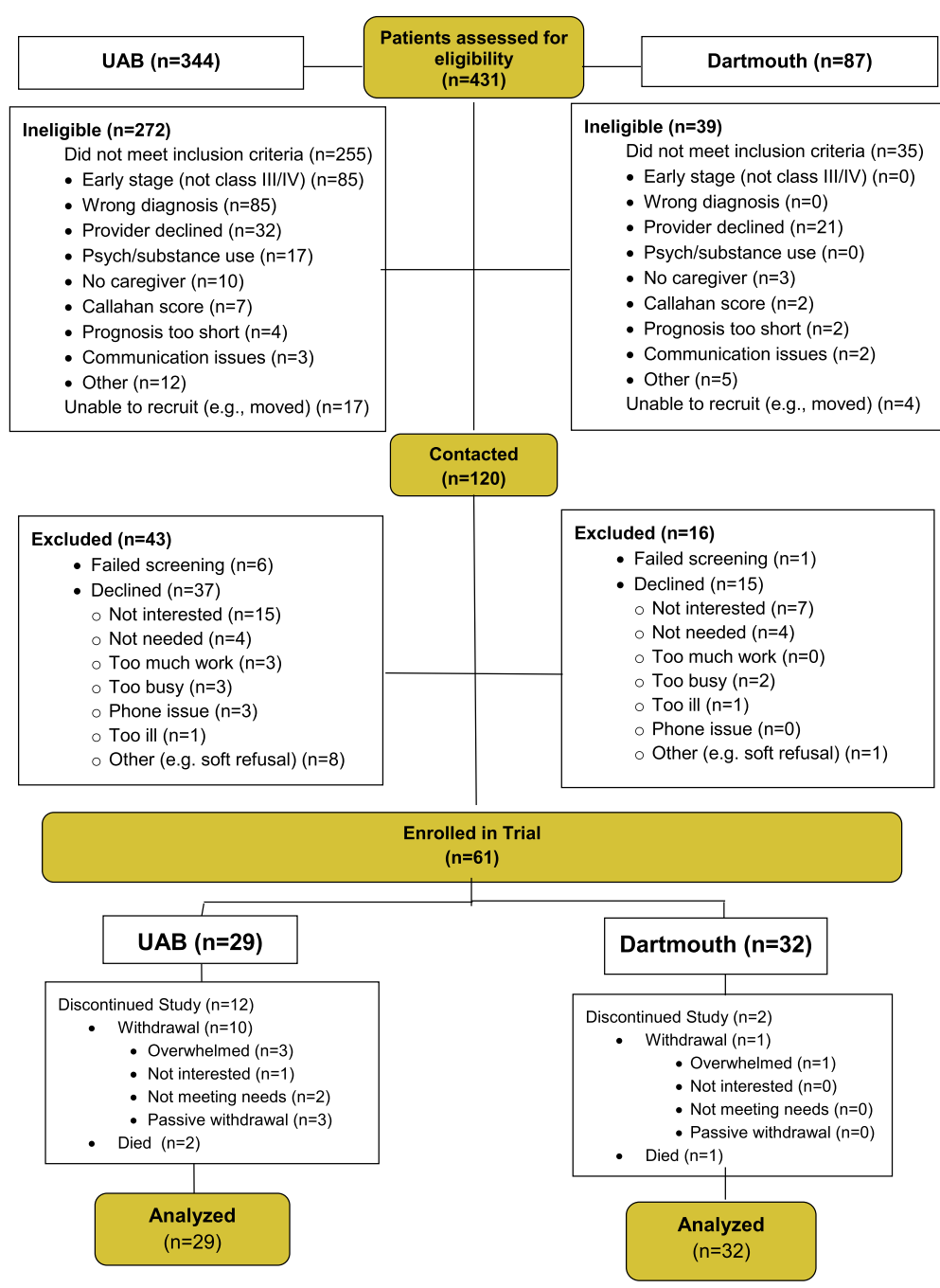

Fig. 2 CONSORT diagram: Patient Recruitment, Treatment, and Analysis 
Table 1 Patient Demographics

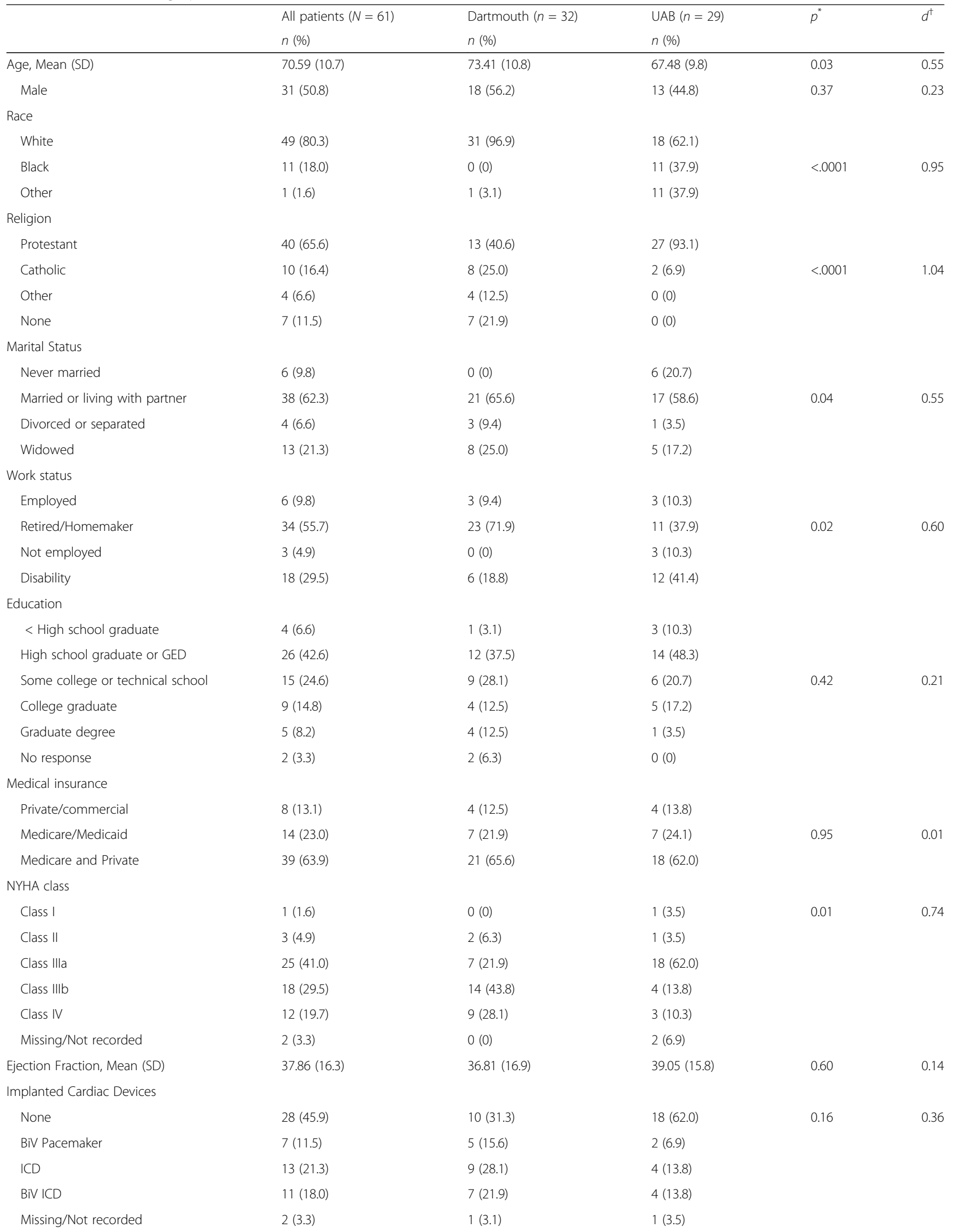


Table 1 Patient Demographics (Continued)

\begin{tabular}{|c|c|c|c|c|c|}
\hline \multicolumn{6}{|l|}{ Heart Medications } \\
\hline ACE-I & $20(32.8)$ & $11(34.4)$ & $9(31.0)$ & 0.78 & 0.07 \\
\hline Beta-blocker & $49(80.3)$ & $29(90.6)$ & $20(69.0)$ & 0.05 & 0.51 \\
\hline ARB & $18(29.5)$ & $13(40.6)$ & $5(17.2)$ & 0.05 & 0.50 \\
\hline Statin & $36(59.0)$ & $17(53.1)$ & $19(65.5)$ & 0.44 & 0.20 \\
\hline Allopurinol & $8(13.1)$ & $5(15.6)$ & $3(10.3)$ & 0.71 & 0.10 \\
\hline Aldosterone blocker & $27(44.3)$ & $17(53.1)$ & $10(34.5)$ & 0.20 & 0.33 \\
\hline None & $3(4.9)$ & $1(3.1)$ & $2(6.9)$ & 0.60 & 0.14 \\
\hline \multicolumn{6}{|l|}{ Diuretic Intake (mg) } \\
\hline Furosemide & $29.02(42.9)$ & $32.19(47.1)$ & $25.52(38.1)$ & 0.55 & 0.16 \\
\hline Bumetanide & $0.03(0.3)$ & $0(0)$ & $0.07(0.4)$ & - & - \\
\hline Torsemide & $25.57(44.0)$ & $36.88(48.9)$ & $24.14(38.6)$ & 0.81 & 0.06 \\
\hline Metolazone & $0.38(1.2)$ & $0(0)$ & $0.72(1.7)$ & - & - \\
\hline Hydrochlorothiazide & $1.02(4.7)$ & $0.78(4.4)$ & $1.28(5.1)$ & 0.69 & 0.11 \\
\hline \multicolumn{6}{|l|}{ Lab data, median } \\
\hline Hemoglobin (g/dL) & $12.3[9-16.4]$ & $12.45[9-16.4]$ & $12.3[10.1-16.2]$ & 0.70 & 0.10 \\
\hline Lymphocyte \%WBC & $16.95[3-48]$ & $22.4[3-48]$ & 10 [4-42] & 0.02 & 0.58 \\
\hline Uric acid (mg/dL) & $6.65[0-11]$ & $7.25[0-11]$ & $2.05[0-7.3]$ & 0.01 & 1.15 \\
\hline Total cholesterol (mg/dL) & $146[0-253]$ & 139 [0-253] & $151[0-259]$ & 0.26 & 0.34 \\
\hline Sodium (mmol/L) & 139 [123-145] & 139.5 [123-145] & 139 [127-145] & 0.78 & 0.07 \\
\hline \multicolumn{6}{|l|}{ Seattle Heart Failure Model } \\
\hline 1-year survival probability, SD & $0.84(0.1)$ & $0.82(0.2)$ & $0.87(0.1)$ & 0.13 & 0.38 \\
\hline 2-year survival probability, SD & $0.72(0.2)$ & $0.69(0.2)$ & $0.76(0.1)$ & 0.14 & 0.39 \\
\hline 5-year survival probability, SD & $0.49(0.2)$ & $0.45(0.2)$ & $0.53(0.2)$ & 0.18 & 0.36 \\
\hline Charlson Comorbidity Index & $6.66(92.5)$ & $6.84(1.8)$ & $6.45(3.0)$ & 0.55 & 0.16 \\
\hline Rural Location & $44(72.1)$ & $31(96.9)$ & $13(44.8)$ & - & - \\
\hline
\end{tabular}

Abbreviations: SD standard deviation, GED General Education Development, NYHA New York Heart Association, BiV biventricular, ICD implantable cardioverterdefibrillator, $A C E-I$ angiotensin-converting-enzyme inhibitor; ARB = angiotensin receptor blocker

" $p$-values from t-test, Chi-squared, or Fisher's exact tests, as appropriate

${ }^{\dagger}$ Effect size: Cohen's $d$ or $d$-equivalent: small: $d \sim 0.2$, medium $d \sim 0.5$, large $d \sim 0.8$

averaged $84 \%$ for 1 -year, $72 \%$ for 2 -years, and $49 \%$ for 5 years.

Family caregivers $(n=48)$ were a mean age of 65 years, female $(81 \%, n=39)$, white $(83 \%, n=40)$, Protestant $(52 \%$, $n=25)$, married or living with a partner $(81 \%, n=39)$, retired $(50 \%, n=24)$, had a graduate degree $(27 \% ; n=13)$ and were the patients' spouse or partner $(65 \%, n=31)$ (Table 2). Compared to DHMC, UAB caregivers were more often black and Protestant.

\section{Feasibility/acceptability: Intervention and measure completion}

Overall study attrition was due to withdrawal $(18 \% ; n=11)$ and death $(5 \% ; n=3)$. In-person comprehensive palliative care assessments were completed by $64 \%(n=39)$ (UAB = $41 \% ; n=12$ vs. DHMC 84.4\%; $n=27)$. Non-completion $(n$ $=22$ ) was due to "declined" (61\%; $n=14)$, "no-show" (26\%; $n=6)$ or died before appointment $(13 \% ; n=2)$. Most patients $(69 \% ; n=42 ; \mathrm{UAB}=41 \% ; n=12$ vs $\mathrm{DHMC}=94 \%$; $n=30)$ and caregivers (79\%; $n=38$; UAB $=63 \% ; n=12$ vs
DHMC $=90 \% ; n=26)$ completed the nurse coaching sessions. Average weekly session duration was 46(caregivers)-50-(patients) minutes and monthly check-in calls were $13 \mathrm{~min}$. At the completion of the weekly sessions, nurse coaches assessed patient and caregiver satisfaction with the intervention, which was high and no participants reported adverse events.

We observed between-site differences in measurement completion for patients $(\mathrm{UAB}=38 \% ; n=11$ vs DHMC $=72 \% ; n=23 ; p=0.008, d$-equivalent $=0.7$ ), but not for caregivers $(\mathrm{UAB}=58 \% ; n=11$ vs $\mathrm{DHMC}=69 \% ; n=20$; $p=0.54, d$-equivalent $=0.18$ ).

Exploratory analyses of study attrition, and participant baseline demographics and outcomes (Additional file 1: Tables S2 and S3) revealed that the strongest predictors of patient attrition were site (UAB vs. DHMC: $\mathrm{OR}=4.7$, 95\% C.I. $=[1.6,14.4], p=0.006)$ and baseline Patient Assessment of Chronic Illness Care (PACIC)-patient activation subscale score $(\mathrm{OR}=0.57$ per $\mathrm{SD}$ increase, 95\% C.I. $=[0.3,0.9], p=0.026)$. The strongest predictor 
Table 2 Caregiver Demographics

\begin{tabular}{|c|c|c|c|c|c|}
\hline & All caregivers $(N=48)$ & Dartmouth $(n=29)$ & $\operatorname{UAB}(n=19)$ & & \\
\hline & $n(\%)$ & $n(\%)$ & $n(\%)$ & $p^{*}$ & $d^{+}$ \\
\hline Age, Mean (SD) & $64.94(9.3)$ & $65.18(10.3)$ & $64.58(7.7)$ & 0.082 & 0.06 \\
\hline \multicolumn{6}{|l|}{ Gender } \\
\hline Female & $39(81.3)$ & $23(79.3)$ & $16(84.2)$ & 0.99 & 0 \\
\hline \multicolumn{6}{|l|}{ Race } \\
\hline White & $40(83.3)$ & $29(100.0)$ & $11(57.9)$ & 0.0002 & 1.19 \\
\hline Black & $8(16.7)$ & $0(0)$ & $8(42.1)$ & & \\
\hline \multicolumn{6}{|l|}{ Religion } \\
\hline Protestant & $25(52.1)$ & $7(24.1)$ & $18(94.7)$ & & \\
\hline Catholic & $7(14.6)$ & $7(24.1)$ & $0(0)$ & & \\
\hline Jewish & $1(2.1)$ & $1(3.5)$ & $0(0)$ & $<.0001$ & 1.26 \\
\hline Other & $6(12.5)$ & $6(20.7)$ & $0(0)$ & & \\
\hline None & $9(18.8)$ & $8(27.6)$ & $1(5.3)$ & & \\
\hline \multicolumn{6}{|l|}{ Marital Status } \\
\hline Never married & $2(4.2)$ & $0(0)$ & $2(10.5)$ & 0.09 & 0.51 \\
\hline Married or living with partner & $39(81.3)$ & $26(89.7)$ & $13(68.4)$ & & \\
\hline Divorced or separated & $3(6.3)$ & $2(6.9)$ & $1(5.3)$ & & \\
\hline Widowed & $4(8.3)$ & $1(3.5)$ & $3(15.8)$ & & \\
\hline \multicolumn{6}{|l|}{ Work status } \\
\hline Employed & $17(35.4)$ & $9(31.0)$ & $8(42.1)$ & & \\
\hline Retired/Homemaker & $24(50.0)$ & $15(51.7)$ & $9(47.4)$ & & \\
\hline Not employed & $3(6.3)$ & $1(3.5)$ & $2(10.5)$ & 0.49 & 0.20 \\
\hline Disability & $3(6.3)$ & $3(10.3)$ & $0(0)$ & & \\
\hline No response & $1(2.1)$ & $1(3.5)$ & $0(0)$ & & \\
\hline \multicolumn{6}{|l|}{ Education } \\
\hline$<$ High school graduate & $3(6.3)$ & $2(6.9)$ & $1(5.3)$ & & \\
\hline High school graduate or GED & $12(25.0)$ & $7(24.1)$ & $5(26.3)$ & & \\
\hline Some college or technical school & $11(22.9)$ & $5(17.2)$ & $6(31.6)$ & 0.23 & 0.36 \\
\hline College graduate & $9(18.8)$ & $4(13.8)$ & $5(26.3)$ & & \\
\hline Graduate degree & $13(27.1)$ & $11(37.9)$ & $2(10.5)$ & & \\
\hline \multicolumn{6}{|l|}{ Relationship to patient } \\
\hline Spouse/partner & $31(64.6)$ & $20(69.0)$ & $11(57.9)$ & & \\
\hline Parent & $8(16.7)$ & $4(13.8)$ & $4(21.1)$ & & \\
\hline Son or daughter & $4(8 . .3)$ & $2(6.9)$ & $2(10.5)$ & 0.63 & 0.14 \\
\hline Other relative & $3(6.3)$ & $1(3.5)$ & $2(10.5)$ & & \\
\hline Friend & $2(4.2)$ & $2(6.9)$ & $0(0)$ & & \\
\hline
\end{tabular}

Abbreviations: SD standard deviation, GED General Education Development

${ }^{*} p$-values from t-test or Fisher's exact tests, as appropriate

${ }^{\dagger}$ Effect size: Cohen's $d$ or $d$-equivalent; small: $d \sim 0.2$, medium $d \sim 0.5$, large $d \sim 0.8$

of caregiver attrition was decreased caregiver QOL (measured by the Bakas Caregiving Outcomes Scale $(\mathrm{BCOS})$ score $)(\mathrm{OR}=0.49$ per $\mathrm{SD}$ increase, $95 \%$ C.I. $=$ $[0.2,1.1], p=0.073)$.

Patient and caregiver feedback relative to the intervention included the density, high literacy level of the patient/caregiver Charting Your Course guides, and difficulty attending (due to travel distance/transportation) and misunderstanding the purpose of the in-person outpatient palliative care consultation. They also provided critical feedback about the study measures: they reported an inability to complete the literacy measure, and a high burden of completing the symptom measure.

\section{Patient reported outcome measures}

Key effect size differences were evident between the UAB and DHMC patient-reported baseline (Additional file 1: Table S4) and change from baseline to week 24 
outcomes (Table 3). At baseline, relative to DHMC, UAB had $>0.20$ effect size differences that were lower in KCCQ symptom subscale, PROMIS mental health, PACIC (activation scale) and humor coping and higher KCCQ-QOL subscale, perceived social support (e.g. MSPSS) and use of denial and religious coping strategies. UAB patients experienced moderate improvements post-intervention (baseline to 24-week) in all KCCQ subscales $(\mathrm{d}=0.37-0.79)$, MSAS-HF symptom burden index, HADS $(\mathrm{d}=0.31-0.34)$, and Physical and Mental Global Health subscales $(d=0.46-0.53)$ and a small magnitude of improvement in the decisionsupport subscale. DHMC patients had a moderate postintervention improvement in the MSAS-HF symptom burden index $(\mathrm{d}=.50)$ and small-moderate improvements (but to a lesser extent than UAB) in the KCCQ $(\mathrm{d}=0.21-0.39)$, HADS $(\mathrm{d}=0.20-0.23)$ and Global Mental Health $(d=0.23)$ subscales.

\section{Caregiver reported outcome measures}

No between-site differences were noted in caregiverreported baseline outcomes (Additional file 1: Table S5).
However post-intervention estimates of change from baseline to 24 weeks demonstrated that UAB caregivers had moderate effect size improvements in BCOS, HADS-Depression, Global Mental Health, and MBCB scores and small magnitude improvements in HADSAnxiety and Global Physical Health (Table 4). At DHMC the moderate post-intervention improvement was noted for MBCB-Stress Burden and MBCB total scores and small improvements in BCOS, HADS-depression and PAC-outlook on life subscales.

\section{Resource use}

At baseline there were no between-group differences in hospital days, intensive care unit (ICU) days or emergency department (ED) visits (Table 5). However, from baseline to 24 weeks, a small-moderate effect size decrease was noted in hospital and ICU days per month; a small effect size decrease in ED visits per month was only noted at UAB. At baseline $87 \%(n=28)$ of DHMC and $28 \%(n=8)$ of UAB patients had an advance directive $(p<0.001)$; by study end, one additional patient per site completed an advance directive. Related to hospice care,

Table 3 Patient-reported Outcome Measures - Change from Baseline (Adjusted)

\begin{tabular}{|c|c|c|c|c|c|c|c|c|c|}
\hline & \multicolumn{3}{|l|}{ All patients } & \multicolumn{3}{|l|}{ Dartmouth } & \multicolumn{3}{|l|}{ UAB } \\
\hline & Mean (SE) & $p^{*}$ & Effect size $^{\dagger}$ & Mean (SE) & $p^{*}$ & Effect size $^{\dagger}$ & Mean (SE) & $p^{*}$ & Effect size $^{\dagger}$ \\
\hline \multicolumn{10}{|l|}{ KCCQ } \\
\hline Physical limitation & $13.30(4.4)$ & 0.003 & 0.50 & $9.90(6.1)$ & 0.11 & 0.37 & $17.90(6.2)$ & 0.01 & 0.67 \\
\hline Symptoms & $10.80(4.3)$ & 0.01 & 0.44 & $5.30(5.5)$ & 0.34 & 0.21 & $17.00(6.6)$ & 0.01 & 0.69 \\
\hline Social limitation & $8.40(5.1)$ & 0.10 & 0.28 & $6.20(6.7)$ & 0.36 & 0.21 & $11.10(7.8)$ & 0.16 & 0.37 \\
\hline Quality of life & $10.70(4.0)$ & 0.009 & 0.4 & $10.40(5.4)$ & 0.06 & 0.39 & $11.30(6.2)$ & 0.07 & 0.42 \\
\hline KCCQ functional status & $11.60(4.0)$ & 0.005 & 0.49 & $6.90(5.3)$ & 0.20 & 0.29 & $17.50(5.8)$ & 0.004 & 0.74 \\
\hline KCCQ clinical summary & $10.30(3.9)$ & 0.009 & 0.44 & $7.80(5.2)$ & 0.14 & 0.33 & $13.70(5.7)$ & 0.02 & 0.58 \\
\hline MSAS-HF Symptom Burden Index & $-25.80(7.1)$ & 0.0004 & -0.54 & $-24.20(8.0)$ & 0.003 & -0.50 & $-25.7(12.8)$ & 0.05 & -0.54 \\
\hline \multicolumn{10}{|l|}{ HADS } \\
\hline Anxiety & $-1.00(0.5)$ & 0.08 & -0.29 & $-0.70(0.6)$ & 0.28 & -0.20 & $-1.20(0.9)$ & 0.20 & -0.34 \\
\hline Depression & $-1.10(0.6)$ & 0.07 & -0.28 & $-0.90(0.7)$ & 0.17 & -0.23 & $-1.20(1.1)$ & 0.28 & -0.31 \\
\hline \multicolumn{10}{|l|}{ PROMIS } \\
\hline Global Physical Health T score & $2.70(1.5)$ & 0.08 & 0.32 & $1.50(2.0)$ & 0.47 & 0.18 & $3.80(2.0)$ & 0.07 & 0.46 \\
\hline Global Mental Health T score & $3.00(1.4)$ & 0.04 & 0.36 & $1.90(1.8)$ & 0.30 & 0.23 & $4.40(2.2)$ & 0.05 & 0.53 \\
\hline \multicolumn{10}{|l|}{ PACIC } \\
\hline Patient activation & $0.20(0.2)$ & 0.26 & 0.17 & $0.20(0.2)$ & 0.35 & 0.17 & $0(0.3)$ & 0.95 & 0 \\
\hline Decision support & $0.30(0.2)$ & 0.10 & 0.30 & $0.20(0.2)$ & 0.45 & 0.2 & $0.30(0.3)$ & 0.23 & 0.30 \\
\hline Goal setting & $0.30(0.2)$ & 0.09 & 0.29 & $0.30(0.2)$ & 0.25 & 0.29 & $0.20(0.2)$ & 0.48 & 0.20 \\
\hline Problem solving & $0.30(0.2)$ & 0.14 & 0.27 & $0.20(0.2)$ & 0.29 & 0.18 & $0.20(0.3)$ & 0.59 & 0.18 \\
\hline Care Coordination & $0(0.2)$ & 0.92 & 0 & $-0.10(0.2)$ & 0.61 & -0.09 & $0.10(0.3)$ & 0.80 & 0.09 \\
\hline PACIC Summary Score & $0.20(0.1)$ & 0.14 & 0.23 & $0.10(0.2)$ & 0.42 & 0.11 & $0.10(0.2)$ & 0.50 & 0.11 \\
\hline
\end{tabular}

Abbreviations: SE standard error, KCCQ Kansas City Cardiomyopathy Questionnaire, MSAS-HF Memorial Symptom Assessment Scale-Heart Failure, HADS Hospital Anxiety and Depression Scale, PROMIS Patient Reported Outcomes Measurement Information System, PACIC Patient Assessment of Chronic IIIness Care. All change from baseline estimates were adjusted for measures associated with attrition (e.g. religious preference, baseline PACIC-Patient Activation subscale and SHFM 1-year survival probability)

" $p$-values from t-test or Fisher's exact tests, as appropriate

${ }^{\dagger}$ Effect size: Cohen's $d$ or $d$-equivalent; small: $d \sim 0.2$, medium $d \sim 0.5$, large $d \sim 0.8$ 
Table 4 Caregiver-reported Outcomes - Change from Baseline to 24 weeks (Adjusted for BCOS)

\begin{tabular}{|c|c|c|c|c|c|c|c|c|c|}
\hline & \multicolumn{3}{|c|}{ All caregivers } & \multicolumn{3}{|l|}{ Dartmouth } & \multicolumn{3}{|l|}{$\cup A B$} \\
\hline & Mean (SE) & $p^{*}$ & Effect size $^{\dagger}$ & Mean (SE) & $p^{*}$ & Effect size $^{\dagger}$ & Mean (SE) & $p^{*}$ & Effect size $^{\dagger}$ \\
\hline BCOS score & $3.70(2.0)$ & 0.07 & 0.40 & $2.30(2.2)$ & 0.30 & 0.25 & $6.70(3.8)$ & 0.09 & 0.73 \\
\hline \multicolumn{10}{|l|}{ HADS } \\
\hline Anxiety & $-0.20(0.5)$ & 0.69 & -0.07 & $0.10(0.7)$ & 0.86 & 0.03 & $-0.70(0.9)$ & 0.42 & -0.23 \\
\hline Depression & $-1.30(0.7)$ & 0.08 & -0.32 & $-1.10(0.9)$ & 0.26 & -0.27 & $-1.90(1.1)$ & 0.11 & -0.47 \\
\hline \multicolumn{10}{|l|}{ PROMIS } \\
\hline Global Physical Health & $1.70(1.4)$ & 0.21 & 0.22 & $1.60(1.8)$ & 0.38 & 0.2 & $1.60(2.2)$ & 0.45 & 0.20 \\
\hline Global Mental Health & $1.80(1.3)$ & 0.18 & 0.24 & $1.00(1.8)$ & 0.57 & 0.13 & $3.20(1.8)$ & 0.08 & 0.43 \\
\hline \multicolumn{10}{|l|}{$\mathrm{MBCB}$} \\
\hline Objective burden & $-1.10(0.5)$ & 0.02 & -0.33 & $-0.50(0.6)$ & 0.39 & -0.15 & $-2.10(0.8)$ & 0.01 & -0.63 \\
\hline Demand burden & $-0.60(0.4)$ & 0.09 & -0.28 & $-0.04(0.3)$ & 0.22 & -0.19 & $-1.20(0.8)$ & 0.16 & -0.56 \\
\hline Stress burden & $-1.30(0.4)$ & 0.001 & -0.58 & $-1.40(0.5)$ & 0.003 & -0.62 & $-1.40(0.7)$ & 0.07 & -0.62 \\
\hline Total Score & $-3.10(1.0)$ & 0.002 & -0.53 & $-2.40(1.0)$ & 0.03 & -0.41 & $-4.60(2.0)$ & 0.03 & -0.78 \\
\hline \multicolumn{10}{|l|}{ PAC } \\
\hline Self-affirmation & $0.40(0.8)$ & 0.58 & 0.11 & $0.30(0.9)$ & 0.70 & 0.08 & $0.60(1.4)$ & 0.68 & 0.16 \\
\hline Outlook on life & $0.40(0.4)$ & 0.41 & 0.16 & $0.50(0.6)$ & 0.41 & 0.20 & $0.40(0.7)$ & 0.61 & 0.16 \\
\hline PAC Total & $0.90(1.2)$ & 0.43 & 0.15 & $0.90(1.4)$ & 0.52 & 0.15 & $1.10(2.0)$ & 0.57 & 0.19 \\
\hline
\end{tabular}

Abbreviations: SE standard error, BCOS Bakas Caregiving Outcomes Scale, HADS Hospital Anxiety and Depression Scale, PROMIS Patient Reported Outcomes Measurement Information System, MBCB Montgomery Borgatta Caregiver Burden Scale, PAC Positive Aspects of Caregiving

" $p$-values from t-test or Fisher's exact tests, as appropriate

${ }^{\dagger}$ Effect size: Cohen's $d$ or $d$-equivalent; small: $d \sim 0.2$, medium $d \sim 0.5$, large $d \sim 0.8$

All change estimates were adjusted for the baseline measures most strongly associated with caregiver attrition: caregiver education, baseline BCOS score and baseline MBCB - objective burden subscale

at baseline each site had one patient enrolled in hospice and by study end, four additional patients were enrolled in hospice $(\mathrm{UAB}=1 ; \mathrm{DHMC}=3)(p=0.07 ; \mathrm{d}=0.48)$.

\section{Discussion}

The purpose of this 2-site, single-arm pilot study was to determine feasibility and potential efficacy of implementing the ENABLE CHF-PC EPC tele-health intervention in a racially-diverse, southeastern US HF population. Previously, ENABLE had demonstrated effectiveness in two large cancer RCTs [33, 43] and in a small, mostly white northeastern HF sample [30]. We were able to achieve our primary study feasibility/acceptability aim in a racially and culturally diverse sample by engaging patients and family caregivers and soliciting their feedback to make improvements in the study design, measures, and intervention.

The key lessons learned from this pilot could be of considerable value to other researchers and clinicians attempting to integrate supportive and palliative care into racially-diverse HF populations. First, health literacy issues were marked in our trial and resulted in changes to future study outcome measures, intervention materials, and recruitment and retention procedures. Per our study coordinator reports, participants expressed frustration and dissatisfaction in completing our original health literacy measure, The Newest Vital Sign [44]. We recommend that others be sensitive to health literacy when working with this population and consider pretesting all measures and materials prior to initiating them in large scale trials.

Second, we encountered significant recruitment challenges in the southeastern site. We needed to screen more UAB individuals for eligibility $(n=344)$ compared to DHMC $(n=87)$ and proportionally fewer eligible $\mathrm{UAB}$ patients agreed to participate. Several factors may explain this discrepancy. The southeast has a high proportion of individuals of black race; this population experiences the highest burden of illness from HF in the US and at a much younger age than whites [45]. Blacks have also been noted to have high rates of healthcare system mistrust and a much lower uptake of palliative and hospice services than whites [46-51]. In response to these factors, we reduced our age eligibility criterion from 65 to 50 years and contracted with a recruitment service who was highly-experienced in community-based research in racially underserved populations and maintained weekly communications with the recruitment service and $\mathrm{UAB}$ cardiologists at HF clinical meetings.

Second, this was our first effort to identify and recruit eligible, racially-diverse HF patients for an early palliative care study. Our cardiologist co-investigators were extremely supportive and cooperative, and assisted us to find the most efficient and effective way to identify eligible patients and refine our screening, recruitment and operational procedures without disrupting clinical 
Table 5 Resource Use At Baseline

\begin{tabular}{|c|c|c|c|c|c|c|c|c|c|}
\hline \multirow[t]{2}{*}{ At Baseline (prior 3 months) } & \multicolumn{3}{|c|}{ All patients $(N=60)$} & \multicolumn{3}{|c|}{ Dartmouth $(n=31)$} & \multicolumn{3}{|c|}{$\mathrm{UAB}(n=29)$} \\
\hline & \multicolumn{3}{|l|}{ Mean (SD) } & \multicolumn{3}{|l|}{ Mean (SD) } & Mean (SD) & $p$ & Effect size \\
\hline Hospital days/month & \multicolumn{3}{|l|}{$1.44(3.0)$} & \multicolumn{3}{|l|}{$1.7(3.6)$} & $1.17(2.2)$ & 0.72 & 0.18 \\
\hline ICU days/month & \multicolumn{3}{|l|}{$0.26(1.0)$} & \multicolumn{3}{|l|}{$0.34(1.3)$} & $0.16(0.6)$ & 0.32 & 0.18 \\
\hline ED visits/month & \multicolumn{3}{|l|}{$0.23(0.4)$} & \multicolumn{3}{|l|}{$0.26(0.4)$} & $0.21(0.4)$ & 0.54 & 0.13 \\
\hline \multicolumn{10}{|c|}{ Resource Use- Difference from Baseline } \\
\hline & \multicolumn{3}{|c|}{ All patients $(N=34)$} & \multicolumn{3}{|c|}{ Dartmouth $(n=23)$} & \multicolumn{3}{|c|}{$\operatorname{UAB}(n=11)$} \\
\hline & Mean (SE) & $p^{*}$ & Effect size $^{\dagger}$ & Mean (SE) & $p^{*}$ & Effect size $e^{\dagger}$ & Mean (SE) & $p^{*}$ & Effect size \\
\hline Hospital days/month & $-0.89(0.3)$ & 0.002 & 0.39 & $-1.18(0.4)$ & 0.006 & 0.57 & $-0.61(0.4)$ & 0.13 & 0.24 \\
\hline Days/month in ICU, Mean & $-0.16(0.1)$ & 0.06 & 0.29 & $-0.12(0.1)$ & 0.23 & 0.26 & $-0.21(0.2)$ & 0.16 & 0.31 \\
\hline ED visits/month, Mean & $-0.05(0.1)$ & 0.33 & 0.17 & $0(0.1)$ & 0.94 & 0.05 & $-0.09(0.1)$ & 0.26 & 0.26 \\
\hline
\end{tabular}

Abbreviations: $S D$ standard deviation, $S E$ standard error, Estimates from longitudinal models fitted with negative binomial distributions (log link), adjusted for baseline, PACIC Patient Activation, and religious preference

* $p$-values from t-test or Fisher's exact tests, as appropriate

${ }^{\dagger}$ Effect size: Cohen's d (Cohen, 1988), or d-equivalent (Rosenthal \& Rubin, 2003) small: $\mathrm{d} \sim 0.2$, moderate $\mathrm{d} \sim 0.5$, large $\mathrm{d} \sim 0.8$

patient care. Early on, we also realized the need to adjust processes to improve UAB participants' uptake of the outpatient palliative care consultation component of the intervention. The UAB Supportive Care and Survivorship Clinic faculty and staff helped us to refine outpatient palliative care consultation scheduling procedures so that, when-ever possible, these visits would coincide with other ap-pointments in an effort to overcome transportation issues of patients who lived long distances from the medical center. Many UAB patients lacked familiarity or had misperceptions about EPC, in some cases confusing it with hospice care. We provided our recruiters with ex-tensive training about the goals of EPC as providing "an extra layer of support" so that they were better able to introduce the study in a non-threatening way. We lever-aged the trusting relationship that most HF patients had with their cardiologists and the UAB health system and carefully 'branded' our materials to be consistent with all other UAB programs. We have also expanded our in-progress RCT recruitment sites to include the local Veteran's Affairs Medical Center, a nurse-led clinic for under-insured HF patients, and we are developing partnerships with community agencies, payers, and churches. Fostering clinical [52] and community $[48,52-54]$ relationships are critical components of successful community-based recruitment.

Third, participant retention was equally challenging. As is common in palliative care trials, $[55,56]$ both sites experienced considerable intervention, measurement and overall attrition. Fewer UAB patients participated in the outpatient palliative care consultation (32\% vs 68\%) and intervention sessions ( $41 \%$ vs $94 \%$ ), and fewer UAB caregivers completed all intervention sessions $(63 \%$ vs 90\%). In exploratory analyses, in addition to site, we found links between patient attrition and lower activation scores and between caregiver attrition and lower QOL scores. We offer two explanations for our differential attrition. First, during joint weekly supervisory meetings, UAB more so than DHMC nurse coaches, reported that participants found the Charting Your Course guides to be lengthy and text-heavy. This combined with lower 'activation' levels may have caused some UAB participants to find the intervention burdensome. We revised our materials to be more colorful and pictorial to address what may have been a health literacy issue. The link between caregiver attrition and quality of life and burden is not surprising given that caregivers have been shown to neglect their own needs in favor of caring for the patient. Hence caregivers may not make time for participation in a support intervention [57]. Of interest, distance to the centers and patients' disability status were not predictors of attrition, reinforcing the ability of telephonic services to increase palliative care access.

Though not powered for hypothesis testing, we identified small-to-moderate longitudinal improvements in QOL, symptom, and psychological patient and caregiverreported outcomes. Of interest is that there was a more robust improvement noted in UAB vs. DHMC patients and caregivers. The higher attrition at UAB may account for this difference. We were also intrigued by the significant between-site differences in the patient activation measure; UAB patients had much lower activation scores than DHMC patients and the scores remained stable over the course of the study. In our prior cancer study we also did not see a signal in this measure [33]. Though others have found improvement in PACIC empowerment subscales from early initiation of supportive care (via nurse navigators) [58] it may be that 'activation' is not the EPC mechanism or that the PACIC is not sufficiently sensitive to detect changes in activation. Alternatively, the relatively high activation scores observed in the DHMC cancer and HF samples, may be an indication that the instrument has a ceiling effect. 
Several limitations of these findings are important to note. First, as a single-arm feasibility pilot study, the trial did not have a control group and was not powered to evaluate efficacy. However, as our primary goal was feasibility in a culturally-diverse clinical setting and population, we learned valuable lessons that informed our subsequent RCT, thereby reinforcing the necessary step of pilot testing interventions prior to embarking on large-scale intervention trials [59]. Second, the patient and caregiver identified post-intervention improvements are inconclusive, preliminary, and not to be generalized as eligibility criteria and intervention changes were made during the course of the pilot. Differential attrition between the two sites may account entirely for the outcome differences. We are currently conducting a large, NIH/NINR-funded clinical trial of ENABLE CHF-PC to evaluate efficacy and address most of these limitations.

\section{Conclusion}

In conclusion, a model of concurrent HF palliative care was feasibly pilot-tested in a heterogeneous sample of individuals with NYHA Class III/IV HF and their family caregivers. Between-site demographic, attrition, and participant-reported outcomes highlight the importance of intervention pilot-testing in culturally-diverse populations.

\section{Additional file}

Additional file 1: Table S1. Description of Primary Outcome Measures. Table S2. Association Between Patient Baseline Characteristic and Attrition ( $N=61)$. Table S3. Association Between Caregiver Baseline Characteristic and Attrition ( $N=48)$. Table S4. Patient-Reported Outcomes- Baseline. Table S5. Caregiver-Reported Outcomes- Baseline. (DOCX $30 \mathrm{~kb}$ )

\section{Acknowledgments}

We would like to thank all of the clinicians and staff of Dartmouth-Hitchcock Medical Center, Department of Cardiology (especially Kathleen MacKay, RN, Virginia Beggs, MSN), Palliative Medicine (Lisa Stephens, MS, APRN, Amelia Cullinan, MD) staff, UAB School of Nursing members (Connie White-Williams, $\mathrm{PhD}, \mathrm{RN}$, Amanda Erba, BSN, RN), UAB Department of Cardiology (especially Renzo Loyaga-Rendon, MD and Deepak Acharya, MD), and UAB Division of Geriatrics, Gerontology, and Palliative Care (especially Rodney Tucker, MD, Ali Ahmed, MD) for supporting the study. We would also like to thank Julie Schach, James Mapson, Cynthia D Johnson, Cynthia Y Johnson, and Lori-Jane Higgins for assisting with recruitment and data collection. Most importantly we thank all patients and caregivers for contributing their time and feedback.

\section{Funding}

This work was supported by a Pilot/Exploratory grant from the National Palliative Care Research Center. Dr. Dionne-Odom has received support from the NIH/ National Institute of Nursing Research (1K99NR015903), the NIH/National Cancer Institute (2R25CA047888-24), the National Palliative Care Research Center, and the American Cancer Society (RSG PCSM-124668).

\section{Availability of data and materials}

The datasets used and/or analysed during the current study are available from the corresponding author on reasonable request.

\section{Authors' contributions}

MB conceived study, oversaw trial design, overall responsibility for trial conduct, data interpretation, wrote main draft of the paper. JNDO oversaw study conduct, assisted with data interpretation and provided substantial contributions to drafting of the manuscript. SVP, JT, EK, KMS assisted with study design, conduct, patient referral, and treatment, reviewed and provided critical feedback and critique of the manuscript. AA developed quantitative analysis protocol, conducted statistical analysis, wrote quantitative analysis section. JF, RW, KK, IA assisted with the acquisition of data and contributed to patient reported qualitative data. DE assisted with data interpretation and analysis. KS assisted with study conception and protocol development. RD assisted with protocol development and study conduct. TS provided day-to-day support in conduct of the study including patient acquisition, data collection and management. ATK co-conceived study and co-developed study protocol, oversaw trial design, and conduct at one site, and assisted with data interpretation. All authors have given their approval of the final version of the manuscript.

\section{Ethics approval and consent to participate}

The study protocol was approved by the institutional review boards of Dartmouth College (Lebanon, New Hampshire) and the University of Alabama at Birmingham (Birmingham, Alabama). All patients and family caregivers provided written informed consent.

\section{Consent for publication}

Not applicable.

\section{Competing interests}

The authors declare that they have no competing interests.

\section{Publisher's Note}

Springer Nature remains neutral with regard to jurisdictional claims in published maps and institutional affiliations.

\section{Author details}

'School of Nursing and Department of Medicine, Division of Gerontology, Geriatrics, and Palliative Care, University of Alabama at Birmingham, Birmingham, AL, USA. ${ }^{2}$ School of Nursing, University of Alabama at Birmingham, 1720 2nd Ave South, MT 412C, Birmingham, AL 35294, USA. ${ }^{3}$ Department of Medicine, Division of Cardiovascular Diseases, University of Alabama at Birmingham, Birmingham, AL, USA. ${ }^{4}$ Department of Medicine, Division of Gerontology, Geriatrics and Palliative Care, University of Alabama at Birmingham, Birmingham, AL, USA. ${ }^{5}$ Cardiology, Dartmouth-Hitchcock Medical Center/Geisel School of Medicine at Dartmouth, Lebanon, NH, USA. ${ }^{6} \mathrm{School}$ of Nursing, University of Alabama at Birmingham, Birmingham, AL, USA. ${ }^{7}$ Faculty of Health Sciences, Nursing Department, Hacettepe University, Ankara, Turkey. ${ }^{8}$ Center for Health Services Research in Primary Care, Durham VA Medical Center, Durham, NC, Division of General Internal Medicine, Department of Medicine, Duke University, Durham, NC, USA. ${ }^{9}$ Department of Medicine, Division of Preventative Medicine, University of Alabama at Birmingham, Birmingham, AL, USA.

Received: 10 April 2017 Accepted: 1 August 2017

Published online: 31 August 2017

\section{References}

1. Mozaffarian D, Benjamin EJ, Go AS, Arnett DK, Blaha MJ, Cushman M, Das SR, de Ferranti S, Després JP, Fullerton HJ, Howard VJ, Huffman MD, Isasi CR, Jiménez MC, Judd SE, Kissela BM, Lichtman JH, Lisabeth LD, Liu S, Mackey RH, Magid DJ, DK MG, Mohler ER, Moy CS, Muntner P, Mussolino ME, Nasir K, Neumar RW, Nichol G, Palaniappan L, Pandey DK, Reeves MJ, Rodriguez CJ, Rosamond W, Sorlie PD, Stein J, Towfighi A, Turan TN, Virani SS, Woo D, Yeh RW, Turner MB, American Heart Association Statistics Committee, Stroke Statistics Subcommittee. Executive summary: heart disease and stroke statistics-2016 update a report from the American Heart Association. Circulation. 2016;133:447-54.

2. Allen LA, Stevenson LW, Grady KL, Goldstein NE, Matlock DD, Arnold RM, Cook NR, Felker GM, Francis GS, Hauptman PJ, Havranek EP, Krumholz HM, Mancini D, Riegel B, Spertus JA, American Heart Association, Council on Quality of Care and Outcomes Research, Council on Cardiovascular Nursing, Council on Clinical Cardiology, Council on Cardiovascular Radiology and 
Intervention, Council on Cardiovascular Surgery and Anesthesia. Decision making in advanced heart failure. Circulation. 2012;125:1928-52.

3. Merlo M, Pivetta A, Pinamonti B, Stolfo D, Zecchin M, Barbati G, Di Lenarda A, Sinagra G. Long-term prognostic impact of therapeutic strategies in patients with idiopathic dilated cardiomyopathy: changing mortality over the last 30 years. Eur J Heart Fail. 2014;16:317-24.

4. Janssen DJ, Spruit MA, Uszko-Lencer NH, Schols JM, Wouters EF. Symptoms, comorbidities, and health care in advanced chronic obstructive pulmonary disease or chronic heart failure. J Palliat Med. 2011;14:735-43.

5. Blinderman CD, Homel P, Billings JA, Portenoy RK, Tennstedt SL. Symptom distress and quality of life in patients with advanced congestive heart failure. J Pain Symptom Manag. 2008;35:594-603.

6. Buck HG, Harkness K, Wion R, Carroll SL, Cosman T, Kaasalainen S, Kryworuchko J, McGillion M, O'Keefe-McCarthy S, Sherifali D, Strachan PH, Arthur HM. Caregivers' contributions to heart failure self-care: a systematic review. Eur J Cardiovasc Nurs. 2015;14(1):79-89. doi:10.1177/1474515113518434. Epub 2014 Jan 6.

7. Doherty LC, Fitzsimons D, Mcllfatrick SJ. Carers' needs in advanced heart failure: A systematic narrative review. Eur J Cardiovasc Nurs. 2016;15(4):20312. doi:10.1177/1474515115585237. Epub 2015 Apr 28.

8. Jaarsma T, Johansson P, Ågren S, Strömberg A. Quality of life and symptoms of depression in advanced heart failure patients and their partners. Curr Opin Support Palliat Care. 2010:4:233-7.

9. Kang X, Li Z, Nolan MT. Informal caregivers' experiences of caring for patients with chronic heart failure: systematic review and metasynthesis of qualitative studies. J Cardiovasc Nurs. 2011;26:386-94.

10. Jessup M, Abraham WT, Casey DE, Feldman AM, Francis GS, Ganiats TG, Konstam MA, Mancini DM, Rahko PS, Silver MA, Stevenson LW, Yancy CW. 2009 focused update: ACCF/AHA guidelines for the diagnosis and Management of Heart Failure in adults: a report of the American College of Cardiology Foundation/American Heart Association task force on practice guidelines: developed in collaboration with the International Society for Heart and Lung Transplantation. Circulation. 2009;1 19:1977-2016.

11. Yancy CW, Jessup M, Bozkurt B, Butler J, Casey DE Jr, Drazner MH, Fonarow GC, Geraci SA, Horwich T, Januzzi JL, Johnson MR, Kasper EK, Levy WC, Masoudi FA, McBride PE, McMurray JJ, Mitchell JE, Peterson PN, Riegel B, Sam F, Stevenson LW, Tang WH, Tsai EJ, Wilkoff BL, American College of Cardiology Foundation, American Heart Association Task Force on Practice Guidelines. 2013 ACCF/AHA guideline for the Management of Heart Failure: a report of the American College of Cardiology Foundation/American Heart Association task force on practice guidelines. J Am Coll Cardiol. 2013;62:e147-239.

12. Goodlin SJ, Hauptman PJ, Arnold R, Grady K, Hershberger RE, Kutner J, Masoudi F, Spertus J, Dracup K, Cleary JF, Medak R, Crispell K, Piña I, Stuart B, Whitney C, Rector T, Teno J, Renlund DG. Consensus statement: palliative and supportive care in advanced heart failure. J Card Fail. 2004;10:200-9.

13. Fang JC, Ewald GA, Allen LA, Butler J, Westlake Canary CA, Colvin-Adams M, Dickinson MG, Levy P, Stough WG, Sweitzer NK, Teerlink JR, Whellan DJ, Albert NM, Krishnamani R, Rich MW, Walsh MN, Bonnell MR, Carson PE, Chan MC, Dries DL, Hernandez AF, Hershberger RE, Katz SD, Moore S, Rodgers JE, Rogers JG, Vest AR, Givertz MM, Heart Failure Society of America Guidelines Committee. Advanced (stage D) heart failure: a statement from the Heart Failure Society of America guidelines committee. J Card Fail. 2015;21:519-34.

14. Adler ED, Goldfinger JZ, Kalman J, Park ME, Meier DE. Palliative care in the treatment of advanced heart failure. Circulation. 2009;120:2597-606.

15. Lindenfeld J, Albert NM, Boehmer JP, Collins SP, Ezekowitz JA, Givertz MM, Katz SD, Klapholz M, Moser DK, Rogers JG, Starling RC, Stevenson WG, Tang WH, Teerlink JR, Walsh MN. HFSA 2010 comprehensive heart failure practice guideline. J Card Fail. 2010;16:e1-194.

16. Beernaert K, Cohen J, Deliens L, Devroey D, Vanthomme K, Pardon K, Van den Block L. Referral to palliative care in COPD and other chronic diseases: a population-based study. Respir Med. 2013;107:1731-9.

17. Lindvall C, Hultman TD, Jackson VA. Overcoming the barriers to palliative care referral for patients with advanced heart failure. J Am Heart Assoc. 2014;3:e000742.

18. Hupcey JE, Kitko L, Alonso W. Palliative Care in Heart Failure. Crit Care Nurs Clin North Am. 2015;27:577-87.

19. Ghashghaei R, Yousefzai R, Adler E. Palliative Care in Heart Failure. Prog Cardiovasc Dis. 2016;58:455-60.

20. Mcllvennan CK, Allen LA. Palliative care in patients with heart failure. BMJ. 2016;353:1010.
21. Davis MP, Temel JS, Balboni T, Glare P. A review of the trials which examine early integration of outpatient and home palliative care for patients with serious illnesses. Ann Palliat Med. 2015;4:99-121.

22. Smith TJ, Temin S, Alesi ER, Abernethy AP, Balboni TA, Basch EM, Ferrell BR, Loscalzo M, Meier DE, Paice JA, Peppercorn JM, Somerfield M, Stovall E, Von Roenn JH. American Society of Clinical Oncology provisional clinical opinion: the integration of palliative care into standard oncology care. J Clin Oncol. 2012;30:880-7.

23. Piccini JP, Berger JS, O'Connor CM. Amiodarone for the prevention of sudden cardiac death: a meta-analysis of randomized controlled trials. Eur Heart J. 2009;30:1245-53.

24. Goldstein NE, Lynn J. Trajectory of end-stage heart failure: the influence of technology and implications for policy change. Perspect Biol Med. 2006;49:10-8.

25. Lynn J, Adamson, DM. Living Well at the End of Life: Adapting Health Care to Serious Chronic Illness in Old age. Santa Monica: RAND: White Paper: 2003

26. Díaz-Toro F, Verdejo HE, Castro PF. Socioeconomic inequalities in heart failure. Heart Fail Clin. 2015;11:507-13.

27. He J, Ogden LG, Bazzano LA, Vupputuri S, Loria C, Whelton PK. Risk factors for congestive heart failure in US men and women: NHANES I epidemiologic follow-up study. Arch Intern Med. 2001;161:996-1002.

28. Kalogeropoulos A, Georgiopoulou V, Kritchevsky SB, Psaty BM, Smith NL, Newman AB, Rodondi N, Satterfield S, Bauer DC, Bibbins-Domingo K, Smith AL, Wilson PW, Vasan RS, Harris TB, Butler J. Epidemiology of incident heart failure in a contemporary elderly cohort: the health, aging, and body composition study. Arch Intern Med. 2009;169:708-15.

29. Kociol RD, Greiner MA, Fonarow GC, Hammill BG, Heidenreich PA, Yancy CW, Peterson ED, Curtis LH, Hernandez AF. Associations of patient demographic characteristics and regional physician density with early physician follow-up among medicare beneficiaries hospitalized with heart failure. Am J Cardiol. 2011;108:985-91.

30. Dionne-Odom JN, Kono A, Frost J, Jackson L, Ellis D, Ahmed A, Azuero A, Bakitas M. Translating and testing the ENABLE: CHF-PC concurrent palliative care model for older adults with heart failure and their family caregivers. J Palliat Med. 2014;17:995-1004.

31. Bakitas M, Lyons KD, Hegel MT, Balan S, Barnett KN, Brokaw FC, Byock IR, Hull JG, Li Z, Mckinstry E. The project ENABLE II randomized controlled trial to improve palliative care for rural patients with advanced cancer: baseline findings, methodological challenges, and solutions. Palliat Support Care. 2009;7(01):75-86.

32. Dionne-Odom JN, Azuero A, Lyons KD, Hull JG, Tosteson T, Li Z, Li Z, Frost J, Dragnev KH, Akyar I, Hegel MT, Bakitas MA. Benefits of early versus delayed palliative care to informal family caregivers of patients with advanced cancer: outcomes from the ENABLE III randomized controlled trial. J Clin Oncol. 2015:33:1446-52.

33. Bakitas MA, Tosteson TD, Li Z, Lyons KD, Hull JG, Li Z, Dionne-Odom JN, Frost J, Dragnev KH, Hegel MT, Azuero A, Ahles TA. Early versus delayed initiation of concurrent palliative oncology care: patient outcomes in the ENABLE III randomized controlled trial. J Clin Oncol. 2015;33:1438-45.

34. Pew Research Center. America's Changing Religious Landscape. 2015. http://www.pewforum.org/2015/05/12/americas-changing-religiouslandscape/.

35. Callahan C, Unverzagt F, Hui S, Perkins A, Hendrie H. Six-item screener to identify cognitive impairment among potential research subjects. Med Care. 2002:40:771-81.

36. National Consensus Project. Clinical practice guidelines for quality palliative care; 3rd Edition,. Brooklyn, NY: National Consensus Project for Quality Palliative Care;2013.

37. Steinhauser KE, Alexander SC, Byock IR, George LK, Olsen MK, Tulsky JA. Do preparation and life completion discussions improve functioning and quality of life in seriously ill patients? Pilot randomized control trial. J Palliat Med. 2008;11:1234-40.

38. Bellg AJ, Borrelli B, Resnick B, Hecht J, Minicucci DS, Ory M, Ogedegbe G, Orwig D, Ernst D, Czajkowski S, Treatment Fidelity Workgroup of the NIH Behavior Change Consortium. Enhancing treatment fidelity in health behavior change studies: best practices and recommendations from the NIH behavior change consortium. Health Psychol. 2004;23:443-51.

39. Harris PA, Taylor R, Thielke R, Payne J, Gonzalez N, Conde JG. Research electronic data capture (REDCap) — a metadata-driven methodology and workflow process for providing translational research informatic support. J Biomed Inform. 2009;42:377-81. 
40. Cohen J. Statistical power analysis for the behavioral sciences. 2nd ed. L. Erlbaum: Hillsdale, New Jersey; 1988.

41. Rosenthal R, Rubin DB. R equivalent: a simple effect size indicator. Psychol Methods. 2003:8:492.

42. Bell ML, Fairclough DL. Practical and statistical issues in missing data for longitudinal patient reported outcomes. Stat Methods Med Res. 2014;23(5): 440-59. doi:10.1177/0962280213476378. Epub 2013 Feb 19.

43. Bakitas MA, Lyons K, Hegel M, Balan S, Brokaw FC, Seville J, Hull JG, Li Z, Tosteson TD, Byock IR, Ahles TA. Effects of a palliative care intervention on clinical outcomes in patients with advanced cancer: the project ENABLE ॥ randomized controlled trial. JAMA. 2009;302:741-9.

44. Weiss B, Mays M, Martz W, Castro KM, DeWalt DA, Pignone MP, Mockbee J, Hale FA. Quick assessment of literacy in primary care: the newest vital sign. Ann Fam Med. 2005;3:514-22.

45. Mujib M, Zhang Y, Feller MA, Ahmed A. Evidence of a "heart failure belt" in the southeastern United States. Am J Cardiol. 2011;107:935-7.

46. Rhodes RL, Elwood B, Lee SC, Tiro JA, Halm EA, Skinner CS. The Desires of Their Hearts: The Multidisciplinary Perspectives of African Americans on End-of-Life Care in the African American Community. Am J Hosp Palliat Care. 2017;34(6):510-7. doi:10.1177/1049909116631776. Epub 2016 Feb 14.

47. Lee JJ, Long AC, Curtis JR, Engelberg RA. The influence of race/ethnicity and education on family ratings of the quality of dying in the ICU. J Pain Symptom Manag. 2016;51:9-16.

48. Johnson J, Hayden T, True J, Simkin D, Colbert L, Thompson B, Stewart D, Martin $L$. The impact of faith beliefs on perceptions of end-of-life care and decision making among African American church members. J Palliat Med. 2016;19:143-8

49. Boucher NA, Raghavan M, Smith A, Arnold R, Johnson KS. Palliative Care in the African American Community \#204. J Palliat Med. 2016;19:228-30.

50. Johnson KS, Kuchibhatla M, Payne R, Tulsky JA. Race and residence: intercounty variation in black-white differences in hospice use. J Pain Symptom Manag. 2013;46:681-90.

51. Drisdom S. Barriers to using palliative care: insight into African American culture. Clin J Oncol Nurs. 2013;17:376-80.

52. Durant RW, Davis RB, St George DM, Williams IC, Blumenthal C, CorbieSmith GM. Participation in research studies: factors associated with failing to meet minority recruitment goals. Ann Epidemiol. 2007;17:634-42.

53. Durant RW, Legedza AT, Marcantonio ER, Freeman MB, Landon BE. Different types of distrust in clinical research among whites and African Americans. J Natl Med Assoc. 2011;103:123-30.

54. Durant RW, Legedza AT, Marcantonio ER, Freeman MB, Landon BE. Willingness to participate in clinical trials among African Americans and whites previously exposed to clinical research. J Cult Divers. 2011;18:8-19.

55. Higginson IJ, Hart S, Burman R, Silber E, Saleem T, Edmonds P. Randomised controlled trial of a new palliative care service: compliance, recruitment and completeness of follow-up. BMC Palliat Care. 2008;7:7.

56. Steinhauser KE, Clipp EC, Hays JC, Olsen M, Arnold R, Christakis NA, Lindquist $\mathrm{JH}$, Tulsky JA. Identifying, recruiting, and retaining seriously-ill patients and their caregivers in longitudinal research. Palliat Med. 2006;20:745-54.

57. Furlong KE, Wuest J. Self-care behaviors of spouses caring for significant others with Alzheimer's disease: the emergence of self-care worthiness as a salient condition. Qual Health Res. 2008;18:1662-72.

58. Wagner EH, Ludman EJ, Aiello Bowles EJ, Penfold R, Reid RJ, Rutter CM, Chubak J, McCorkle R. Nurse navigators in early cancer care: a randomized, controlled trial. J Clin Oncol. 2014;32:12-8.

59. Medical Research Council. Developing and evaluating complex interventions: new guidance. London: Medical Research Council; 2016.

\section{Submit your next manuscript to BioMed Central and we will help you at every step:}

- We accept pre-submission inquiries

- Our selector tool helps you to find the most relevant journal

- We provide round the clock customer support

- Convenient online submission

- Thorough peer review

- Inclusion in PubMed and all major indexing services

- Maximum visibility for your research

Submit your manuscript at www.biomedcentral.com/submit
) Biomed Central 